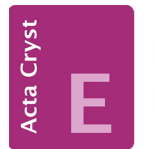

ISSN 2056-9890

\section{Crystal structure of $\left(5^{\prime} S, 8^{\prime} S\right)$-3-(2,5-di- methylphenyl)-8-methoxy-3-nitro-1-aza- spiro[4.5]decane-2,4-dione}

\section{Gao-Bo Hu, Da-Wei Jiang, Jiang-Yan Li, Yan Rao and Li-Yuan Jiang*}

Medical College, Quzhou College of Technology, Quzhou 324000, People's Republic of China. *Correspondence e-mail: jiangly1205@163.com

Received 15 December 2014; accepted 7 March 2015

Edited by W. T. A. Harrison, University of Aberdeen, Scotland

The title compound, $\mathrm{C}_{18} \mathrm{H}_{22} \mathrm{~N}_{2} \mathrm{O}_{5}$, was synthesized by nitrification of its enol precursor. The pyrrolidine ring plane adopts a twisted conformation about the $\mathrm{C}-\mathrm{C}$ bond linking the spiro centre and the $\mathrm{C}=\mathrm{O}$ group remote from the $\mathrm{N}$ atom. It makes dihedral angles of $71.69(9)$ and $88.92(9)^{\circ}$, respectively, with the benzene ring plane and the plane defined by the four $\mathrm{C}$ atoms that form the seat of the of the cyclohexane chair. At the spiro centre, the $\mathrm{NH}$ group is axial and the $\mathrm{C}=\mathrm{O}$ group is equatorial with respect to the cyclohexane ring. In the crystal, inversion dimers linked by pairs of $\mathrm{N}-\mathrm{H} \cdots \mathrm{O}$ hydrogen bonds generate $R_{2}^{2}(8)$ loops. The dimers are linked by $\mathrm{C}-\mathrm{H} \cdots \mathrm{O}$ interactions, generating a three-dimensional network.

Keywords: crystal structure; 1-azaspiro[4.5]decane-2,4-dione; hydrogen bonding; pesticide; spirotetramat.

CCDC reference: 1052631

\section{Related literature}

For the pesticide spirotetramat, the central unit of the title compound, see: Fischer \& Weiss (2008); Maus (2008); Bruck et al. (2009); Campbell et al. (1985); Schobert \& Schlenk (2008). For structures of spirotetramat derivatives, see: Fischer et al. (2010).

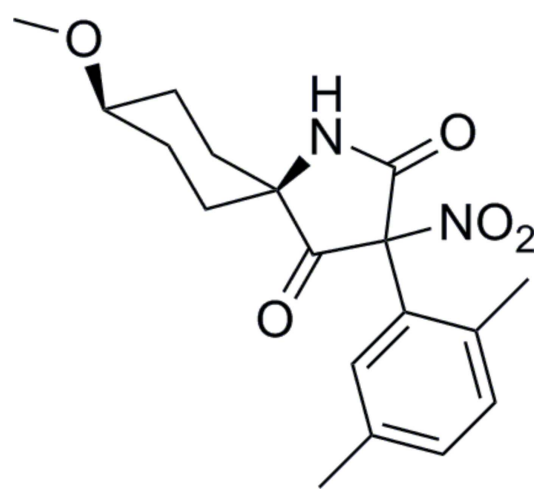

2. Experimental

2.1. Crystal data

$\mathrm{C}_{18} \mathrm{H}_{22} \mathrm{~N}_{2} \mathrm{O}_{5}$

$M_{r}=346.38$

Monoclinic, $P 2_{1} / c$

$a=9.5707$ (9) А

$b=8.4181(7) \AA$

$c=22.8720$ (19) $\AA$

$\beta=100.703(8)^{\circ}$

$$
\begin{aligned}
& V=1810.7(3) \AA^{3} \\
& Z=4 \\
& \text { Mo } K \alpha \text { radiation } \\
& \mu=0.09 \mathrm{~mm}^{-1} \\
& T=170 \mathrm{~K} \\
& 0.36 \times 0.32 \times 0.23 \mathrm{~mm}
\end{aligned}
$$

\subsection{Data collection}

Agilent Xcalibur (Atlas, Gemini ultra) diffractometer

Absorption correction: multi-scan (CrysAlis PRO; Agilent, 2011)

$T_{\min }=0.954, T_{\max }=1.000$

6891 measured reflections 3308 independent reflections 2600 reflections with $I>2 \sigma(I)$ $R_{\text {int }}=0.034$

\subsection{Refinement}

$R\left[F^{2}>2 \sigma\left(F^{2}\right)\right]=0.050$

$w R\left(F^{2}\right)=0.139$

$S=1.04$

3308 reflections
229 parameters

$\mathrm{H}$-atom parameters constrained

$\Delta \rho_{\max }=0.60{\mathrm{e} \AA^{-3}}^{-3}$

$\Delta \rho_{\min }=-0.31{\mathrm{e} \AA^{-3}}^{-3}$
Table 1

Hydrogen-bond geometry $\left(\AA{ }^{\circ}\right)$.

\begin{tabular}{lllll}
\hline$D-\mathrm{H} \cdots A$ & $D-\mathrm{H}$ & $\mathrm{H} \cdots A$ & $D \cdots A$ & $D-\mathrm{H} \cdots A$ \\
\hline $\mathrm{N} 2-\mathrm{H} 2 \cdots \mathrm{O} 3^{\mathrm{i}}$ & 0.88 & 2.02 & $2.8853(19)$ & 167 \\
$\mathrm{C} 4-\mathrm{H} 4 \cdots 5^{\text {ii }}$ & 0.95 & 2.57 & $3.287(3)$ & 132 \\
$\mathrm{C} 7-\mathrm{H} 7 B \cdots \mathrm{O} 1^{\text {ii }}$ & 0.98 & 2.49 & $3.454(3)$ & 168 \\
$\mathrm{C} 14-\mathrm{H} 14 B \cdots \mathrm{O} 2^{\text {iv }}$ & 0.99 & 2.54 & $3.265(3)$ & 130 \\
\hline
\end{tabular}

Symmetry codes: (i) $-x+1,-y+2,-z+1$; (ii) $x,-y+\frac{3}{2}, z-\frac{1}{2}$; (iii) $-x, y-\frac{1}{2},-z+\frac{1}{2}$; (iv) $-x,-y+2,-z+1$.

Data collection: CrysAlis PRO (Agilent, 2011); cell refinement: CrysAlis PRO; data reduction: CrysAlis PRO; program(s) used to solve structure: SHELXS97 (Sheldrick, 2008); program(s) used to refine structure: SHELXL97 (Sheldrick, 2008); molecular graphics: OLEX2 (Dolomanov et al., 2009); software used to prepare material for publication: $O L E X 2$.

Supporting information for this paper is available from the IUCr electronic archives (Reference: HB7342). 


\section{data reports}

\section{References}

Brück, E., Elbert, A., Fischer, R., Krueger, S., Kühnhold, J., Klueken, A. M., Nauen, R., Niebes, J. F., Reckmann, U., Schnorbach, H. J., Steffens, R. \& van Waetermeulen, X. (2009). Crop Prot. 28, 838-844.

Campbell, A. C., Maidment, M. S., Pick, J. H. \& Stevenson, D. F. M. (1985). J. Chem. Soc. Perkin Trans. 1, p. 1567.

Dolomanov, O. V., Bourhis, L. J., Gildea, R. J., Howard, J. A. K. \& Puschmann, H. (2009). J. Appl. Cryst. 42, 339-341.
Fischer, R., Bretschneider, T., Lehr, S., Arnold, C., Dittgen, J., Feucht, D., Kehne, H., Malsam, O., Rosinger, C. H., Franken, E. M. \& Goergens, U. (2010). US Patent No. 20100279873A1.

Fischer, R. \& Weiss, H. C. (2008). Bayer CropSci. J. 61(2), 127-140.

Maus, C. (2008). Bayer CropSci. J. 61, 159-180.

Schobert, R. \& Schlenk, A. (2008). Bioorg. Med. Chem. 16, 4203-4221.

Sheldrick, G. M. (2008). Acta Cryst. A64, 112-122. 


\section{supporting information}

Acta Cryst. (2015). E71, o238-o239 [doi:10.1107/S2056989015004715]

\section{Crystal structure of (5'S,8'S)-3-(2,5-dimethylphenyl)-8-methoxy-3-nitro-1-aza- spiro[4.5]decane-2,4-dione}

\section{Gao-Bo Hu, Da-Wei Jiang, Jiang-Yan Li, Yan Rao and Li-Yuan Jiang}

\section{S1. Comment}

Spirotetramat is a new systemic insecticide which chemically belongs to the class of spirocyclic tetramic acid derivatives and be developed by Bayer CropScience AG (Fischer et al., 2008; Maus, 2008). A unique mode of action coupled with a high degree of activity on targeted pests and low toxicity to nontarget organisms make spirocyclic tetronic acid compounds as a new tool for integrated pest management (Bruck et al., 2009; Campbell et al.,1985; Schobert et al., 2008) In order to study the influence of new substituents on the activity of the Spirotetramat derivative, the title compound, has been synthesized and its structure has been determined (Fig. 1). The molecule contains one benzene ring, one six membered ring, and one five membered ring. The cyclohexane ring adopts a chair conformation; the $\mathrm{C} 13, \mathrm{C} 14$, $\mathrm{C} 16$ and $\mathrm{C} 17$ atoms are on one plane with $\mathrm{C} 15$ and $\mathrm{C} 11$ deviating by 0.658 (5)and -0.676 (9) $\AA$, respectively. There are three planes in the molecule: atoms of $\mathrm{C} 10, \mathrm{C} 11, \mathrm{C} 12$ and $\mathrm{N} 2$ generate the pyrrolidine plane (I), $\mathrm{C} 1-\mathrm{C} 6$ yield the benzene plane (II) and $\mathrm{C} 13-\mathrm{C} 14$ and $\mathrm{C} 16-\mathrm{C} 17$ form the cyclohexane plane(III). The angle between planes I and II is $71.69(9)^{\circ}$, and that between planes I and III is $88.92(9)^{\circ}$. The space arrangement might result from the space factor between groups.

\section{S2. Experimental}

A solution of fuming nitric acid $(0.92 \mathrm{~g}, 16 \mathrm{mmoL})$ in anhydrous chloroform $(10 \mathrm{ml})$ was added dropwise to a solution of compound $2(1.78 \mathrm{~g}, 5.9 \mathrm{mmoL})$ in anhydrous chloroform $(20 \mathrm{ml})$ at 0 degree and stirred for $2 \mathrm{~h}$. The reaction mixture was then washed with ice water $(15 \mathrm{ml})$, and saturated sodium chloride solution and dried over anhydrous $\mathrm{Na}_{2} \mathrm{SO}_{4}$. The solvent was evaporated, and the residual solid was crystallized from ethanol to afford $1.84 \mathrm{~g}$ compound 3 as a pale yellow solid: yield 90\%. The 1H NMR, 13 C-NMR and ESI-MS data testified the title compound's structure. ESI-MS: 347 $(M+\mathrm{H})+(100 \%) ; 1 \mathrm{H}$ NMR (500 MHz, CDCl3): $7.48(\mathrm{~s}, 1 \mathrm{H},-\mathrm{NH}-), 7.55(\mathrm{~s}, 1 \mathrm{H}, \mathrm{Ph}-\mathrm{H}), 7.14(\mathrm{~d}, 1 \mathrm{H}, \mathrm{Ph}-\mathrm{H}), 7.07(\mathrm{~d}$, $1 \mathrm{H}, \mathrm{Ph}-\mathrm{H}), 3.54$ (s, 3H, -OCH3), 3.34-3.33 (m, 1H, $-\mathrm{CH}-\mathrm{O}-\mathrm{C}-$ ), 2.36 (s, 3H, Ph-Me), 2.27 (s, 3H, Ph-Me), 2.12-2.07 (m, 4H, Cyclohexane-H4), 1.99-1.56 (m, 4H, Cyclohexane-H4); 13 C-NMR (100 MHz, CDCl3): 199.9, 165.1, 136.7, 136.0, 133.2, 131.5, 129.7, 127.4, 95.8, 76.8, 75.6, 66.1, 55.7, 32.5, 31.5, 26.4, 26.3, 21.0, 20.3.

\section{S3. Refinement}

The $\mathrm{H}$ atoms were geometrically placed $(\mathrm{C}-\mathrm{H}=0.93-0.98 \AA)$ and refined as riding with $U_{\text {iso }}(\mathrm{H})=1.2 U_{\mathrm{eq}}(\mathrm{C})$. 


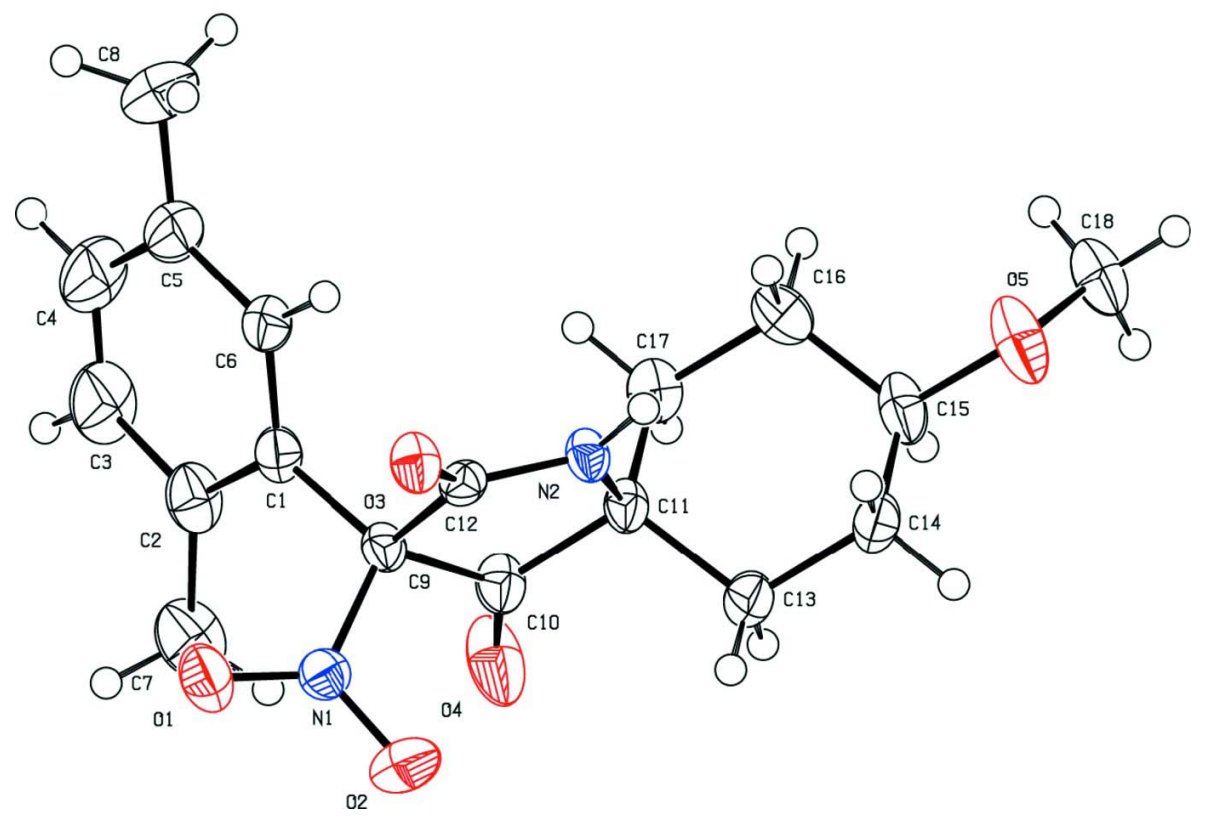

Figure 1

The molecular structure of title molecule, showing 50\% displacement ellipsoids.

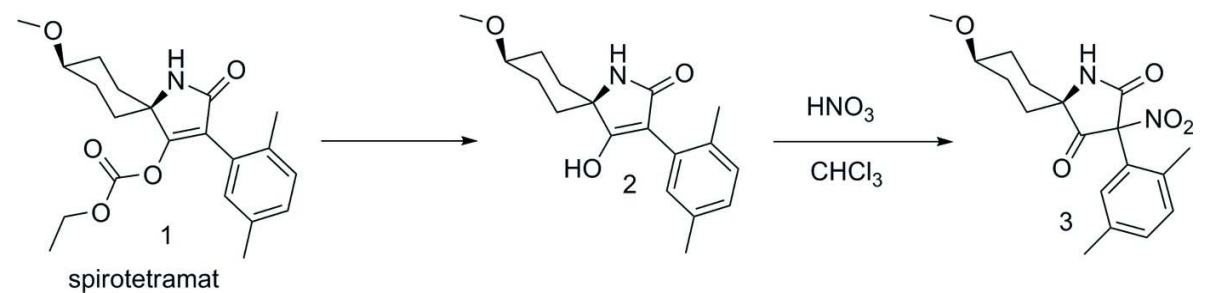

Figure 2

Reaction scheme.

(5'S,8'S)-3-(2,5-Dimethylphenyl)-8-methoxy-3-nitro-1-azaspiro[4.5]decane-2,4-dione

\section{Crystal data}

$\mathrm{C}_{18} \mathrm{H}_{22} \mathrm{~N}_{2} \mathrm{O}_{5}$

$M_{r}=346.38$

Monoclinic, $P 2_{1} / c$

$a=9.5707(9) \AA$

$b=8.4181(7) \AA$

$c=22.8720(19) \AA$

$\beta=100.703(8)^{\circ}$

$V=1810.7(3) \AA^{3}$

$Z=4$

\section{Data collection}

Agilent Xcalibur (Atlas, Gemini ultra) diffractometer

Radiation source: Enhance (Mo) X-ray Source Graphite monochromator

Detector resolution: 10.3592 pixels $\mathrm{mm}^{-1}$
$F(000)=736$

$D_{\mathrm{x}}=1.271 \mathrm{Mg} \mathrm{m}^{-3}$

Mo $K \alpha$ radiation, $\lambda=0.71073 \AA$

Cell parameters from 2205 reflections

$\theta=3.2-29.5^{\circ}$

$\mu=0.09 \mathrm{~mm}^{-1}$

$T=170 \mathrm{~K}$

Block, colourless

$0.36 \times 0.32 \times 0.23 \mathrm{~mm}$

$\omega$ scans

Absorption correction: multi-scan

(CrysAlis PRO; Agilent, 2011)

$T_{\text {min }}=0.954, T_{\max }=1.000$

6891 measured reflections 
3308 independent reflections

2600 reflections with $I>2 \sigma(I)$

$R_{\text {int }}=0.034$

$\theta_{\max }=25.4^{\circ}, \theta_{\min }=3.3^{\circ}$

\section{Refinement}

Refinement on $F^{2}$

Least-squares matrix: full

$R\left[F^{2}>2 \sigma\left(F^{2}\right)\right]=0.050$

$w R\left(F^{2}\right)=0.139$

$S=1.04$

3308 reflections

229 parameters

0 restraints

Primary atom site location: structure-invariant direct methods $h=-11 \rightarrow 7$

$k=-8 \rightarrow 10$

$l=-22 \rightarrow 27$

Secondary atom site location: difference Fourier map

Hydrogen site location: inferred from neighbouring sites

$\mathrm{H}$-atom parameters constrained

$w=1 /\left[\sigma^{2}\left(F_{\mathrm{o}}^{2}\right)+(0.0602 P)^{2}+0.8521 P\right]$

where $P=\left(F_{\mathrm{o}}^{2}+2 F_{\mathrm{c}}^{2}\right) / 3$

$(\Delta / \sigma)_{\max }<0.001$

$\Delta \rho_{\max }=0.60 \mathrm{e} \AA^{-3}$

$\Delta \rho_{\min }=-0.31$ e $\AA^{-3}$

\section{Special details}

Experimental. Absorption correction: CrysAlisPro, Agilent Technologies, Version 1.171.35.11 (release 16-05-2011 CrysAlis171 .NET) (compiled May 16 2011,17:55:39) Empirical absorption correction using spherical harmonics, implemented in SCALE3 ABSPACK scaling algorithm.

Geometry. All e.s.d.'s (except the e.s.d. in the dihedral angle between two 1.s. planes) are estimated using the full covariance matrix. The cell e.s.d.'s are taken into account individually in the estimation of e.s.d.'s in distances, angles and torsion angles; correlations between e.s.d.'s in cell parameters are only used when they are defined by crystal symmetry. An approximate (isotropic) treatment of cell e.s.d.'s is used for estimating e.s.d.'s involving 1.s. planes.

Refinement. Refinement of $F^{2}$ against ALL reflections. The weighted $R$-factor $w R$ and goodness of fit $S$ are based on $F^{2}$, conventional $R$-factors $R$ are based on $F$, with $F$ set to zero for negative $F^{2}$. The threshold expression of $F^{2}>\sigma\left(F^{2}\right)$ is used only for calculating $R$-factors (gt) etc. and is not relevant to the choice of reflections for refinement. $R$-factors based on $F^{2}$ are statistically about twice as large as those based on $F$, and $R$ - factors based on ALL data will be even larger.

Fractional atomic coordinates and isotropic or equivalent isotropic displacement parameters $\left(\AA^{2}\right)$

\begin{tabular}{lllll}
\hline & $x$ & $y$ & $z$ & $U_{\text {iso }} * / U_{\text {eq }}$ \\
\hline O1 & $0.18294(17)$ & $1.31285(17)$ & $0.34437(6)$ & $0.0406(4)$ \\
O2 & $0.09810(17)$ & $1.2327(2)$ & $0.42014(7)$ & $0.0500(5)$ \\
O3 & $0.44250(14)$ & $1.14145(16)$ & $0.43813(5)$ & $0.0295(3)$ \\
O4 & $-0.00240(16)$ & $0.9051(2)$ & $0.37430(8)$ & $0.0579(5)$ \\
O5 & $0.32239(19)$ & $0.4533(2)$ & $0.56929(7)$ & $0.0558(5)$ \\
N1 & $0.16126(17)$ & $1.2114(2)$ & $0.37880(7)$ & $0.0281(4)$ \\
N2 & $0.33749(16)$ & $0.90936(19)$ & $0.45886(6)$ & $0.0245(4)$ \\
H2 & 0.4057 & 0.8790 & 0.4880 & $0.029^{*}$ \\
C1 & $0.2650(2)$ & $1.0141(2)$ & $0.31529(8)$ & $0.0277(4)$ \\
C2 & $0.1642(2)$ & $1.0202(3)$ & $0.26206(9)$ & $0.0381(5)$ \\
C3 & $0.2143(3)$ & $0.9836(3)$ & $0.21029(10)$ & $0.0558(7)$ \\
H3 & 0.1491 & 0.9859 & 0.1735 & $0.067 *$ \\
C4 & $0.3526(3)$ & $0.9447(3)$ & $0.20978(11)$ & $0.0612(8)$ \\
H4 & 0.3801 & 0.9187 & 0.1731 & $0.073^{*}$ \\
C5 & $0.4534(3)$ & $0.9422(3)$ & $0.26167(10)$ & $0.0483(6)$ \\
C6 & $0.4058(2)$ & $0.9761(2)$ & $0.31421(9)$ & $0.0342(5)$ \\
H6 & 0.4721 & 0.9732 & 0.3507 & $0.041^{*}$ \\
C7 & $0.0114(3)$ & $1.0702(3)$ & $0.25703(10)$ & $0.0509(7)$
\end{tabular}




$\begin{array}{lllll}\text { H7A } & -0.0125 & 1.0761 & 0.2968 & 0.076^{*} \\ \text { H7B } & -0.0505 & 0.9924 & 0.2330 & 0.076^{*} \\ \text { H7C } & -0.0024 & 1.1747 & 0.2379 & 0.076^{*} \\ \text { C8 } & 0.6086(3) & 0.9084(4) & 0.26190(13) & 0.0732(9) \\ \text { H8A } & 0.6263 & 0.7941 & 0.2671 & 0.110^{*} \\ \text { H8B } & 0.6674 & 0.9663 & 0.2947 & 0.110^{*} \\ \text { H8C } & 0.6327 & 0.9428 & 0.2241 & 0.110^{*} \\ \text { C9 } & 0.22283(19) & 1.0470(2) & 0.37501(8) & 0.0237(4) \\ \text { C10 } & 0.1216(2) & 0.9211(2) & 0.39383(8) & 0.0305(5) \\ \text { C11 } & 0.20633(19) & 0.8179(2) & 0.44258(8) & 0.0258(4) \\ \text { C12 } & 0.34921(19) & 1.0401(2) & 0.42782(8) & 0.0228(4) \\ \text { C13 } & 0.1293(2) & 0.8034(3) & 0.49537(9) & 0.0346(5) \\ \text { H13A } & 0.1177 & 0.9104 & 0.5118 & 0.042^{*} \\ \text { H13B } & 0.0334 & 0.7582 & 0.4815 & 0.042^{*} \\ \text { C14 } & 0.2111(2) & 0.6980(3) & 0.54417(9) & 0.0374(5) \\ \text { H14A } & 0.3025 & 0.7494 & 0.5613 & 0.045^{*} \\ \text { H14B } & 0.1555 & 0.6857 & 0.5763 & 0.045^{*} \\ \text { C15 } & 0.2394(2) & 0.5373(3) & 0.52057(9) & 0.0384(5) \\ \text { H15 } & 0.1470 & 0.4806 & 0.5074 & 0.046^{*} \\ \text { C16 } & 0.3164(2) & 0.5505(2) & 0.46846(9) & 0.0349(5) \\ \text { H16A } & 0.3296 & 0.4430 & 0.4527 & 0.042^{*} \\ \text { H16B } & 0.4117 & 0.5974 & 0.4823 & 0.042^{*} \\ \text { C17 } & 0.2336(2) & 0.6532(2) & 0.41893(9) & 0.0328(5) \\ \text { H17A } & 0.1417 & 0.6015 & 0.4026 & 0.039^{*} \\ \text { H17B } & 0.2882 & 0.6635 & 0.3864 & 0.039^{*} \\ \text { C18 } & 0.3060(3) & 0.2894(3) & 0.56796(12) & 0.0524(7) \\ \text { H18A } & 0.3302 & 0.2475 & 0.5311 & 0.079^{*} \\ \text { H18B } & 0.2071 & 0.2627 & 0.5696 & 0.079^{*} \\ \text { H18C } & 0.3691 & 0.2424 & 0.6023 & 0.079^{*} \\ & & & & \end{array}$

Atomic displacement parameters $\left(\AA^{2}\right)$

\begin{tabular}{lllllll}
\hline & $U^{11}$ & $U^{22}$ & $U^{33}$ & $U^{12}$ & $U^{13}$ & $U^{23}$ \\
\hline O1 & $0.0575(10)$ & $0.0297(8)$ & $0.0332(8)$ & $0.0042(7)$ & $0.0051(7)$ & $0.0052(7)$ \\
O2 & $0.0483(10)$ & $0.0597(11)$ & $0.0476(9)$ & $0.0133(8)$ & $0.0233(8)$ & $-0.0020(8)$ \\
O3 & $0.0273(7)$ & $0.0311(7)$ & $0.0276(7)$ & $-0.0056(6)$ & $-0.0009(5)$ & $0.0041(6)$ \\
O4 & $0.0303(9)$ & $0.0716(12)$ & $0.0634(11)$ & $-0.0161(8)$ & $-0.0136(8)$ & $0.0371(10)$ \\
O5 & $0.0640(12)$ & $0.0480(10)$ & $0.0476(10)$ & $-0.0009(8)$ & $-0.0099(8)$ & $0.0225(8)$ \\
N1 & $0.0251(9)$ & $0.0339(10)$ & $0.0245(8)$ & $0.0026(7)$ & $0.0021(7)$ & $0.0004(8)$ \\
N2 & $0.0227(8)$ & $0.0274(9)$ & $0.0214(8)$ & $-0.0010(7)$ & $-0.0009(6)$ & $0.0055(7)$ \\
C1 & $0.0367(11)$ & $0.0236(10)$ & $0.0228(9)$ & $-0.0002(8)$ & $0.0052(8)$ & $0.0010(8)$ \\
C2 & $0.0505(14)$ & $0.0352(12)$ & $0.0253(10)$ & $-0.0028(10)$ & $-0.0015(9)$ & $-0.0014(9)$ \\
C3 & $0.084(2)$ & $0.0578(16)$ & $0.0226(11)$ & $0.0047(15)$ & $0.0014(11)$ & $-0.0071(11)$ \\
C4 & $0.093(2)$ & $0.0641(18)$ & $0.0323(13)$ & $0.0112(16)$ & $0.0262(14)$ & $-0.0061(12)$ \\
C5 & $0.0671(17)$ & $0.0443(14)$ & $0.0393(13)$ & $0.0146(12)$ & $0.0247(12)$ & $0.0037(11)$ \\
C6 & $0.0424(12)$ & $0.0349(11)$ & $0.0267(10)$ & $0.0065(10)$ & $0.0106(9)$ & $0.0048(9)$ \\
C7 & $0.0493(15)$ & $0.0596(16)$ & $0.0350(12)$ & $-0.0003(12)$ & $-0.0145(10)$ & $-0.0009(12)$ \\
C8 & $0.076(2)$ & $0.089(2)$ & $0.0660(18)$ & $0.0305(18)$ & $0.0426(16)$ & $0.0096(17)$
\end{tabular}


supporting information

\begin{tabular}{lllllll}
\hline C9 & $0.0233(10)$ & $0.0254(10)$ & $0.0212(9)$ & $0.0009(8)$ & $0.0009(7)$ & $0.0036(8)$ \\
C10 & $0.0258(11)$ & $0.0363(11)$ & $0.0275(10)$ & $-0.0049(9)$ & $0.0003(8)$ & $0.0060(9)$ \\
C11 & $0.0215(10)$ & $0.0308(10)$ & $0.0237(9)$ & $-0.0033(8)$ & $0.0011(7)$ & $0.0057(8)$ \\
C12 & $0.0207(9)$ & $0.0286(10)$ & $0.0192(9)$ & $0.0010(8)$ & $0.0039(7)$ & $-0.0007(8)$ \\
C13 & $0.0297(11)$ & $0.0420(12)$ & $0.0340(11)$ & $0.0025(9)$ & $0.0106(9)$ & $0.0106(10)$ \\
C14 & $0.0338(12)$ & $0.0508(14)$ & $0.0294(11)$ & $0.0028(10)$ & $0.0108(9)$ & $0.0135(10)$ \\
C15 & $0.0332(12)$ & $0.0409(13)$ & $0.0367(11)$ & $-0.0065(10)$ & $-0.0047(9)$ & $0.0161(10)$ \\
C16 & $0.0354(12)$ & $0.0279(11)$ & $0.0381(12)$ & $0.0004(9)$ & $-0.0021(9)$ & $-0.0013(9)$ \\
C17 & $0.0348(12)$ & $0.0326(11)$ & $0.0289(10)$ & $-0.0060(9)$ & $0.0000(8)$ & $-0.0008(9)$ \\
C18 & $0.0645(17)$ & $0.0363(13)$ & $0.0544(15)$ & $0.0038(12)$ & $0.0059(12)$ & $0.0190(12)$ \\
\hline
\end{tabular}

Geometric parameters $\left(\AA,{ }^{\circ}\right)$

\begin{tabular}{|c|c|c|c|}
\hline $\mathrm{O} 1-\mathrm{N} 1$ & $1.206(2)$ & $\mathrm{C} 8-\mathrm{H} 8 \mathrm{~A}$ & 0.9800 \\
\hline $\mathrm{O} 2-\mathrm{N} 1$ & $1.226(2)$ & $\mathrm{C} 8-\mathrm{H} 8 \mathrm{~B}$ & 0.9800 \\
\hline $\mathrm{O} 3-\mathrm{C} 12$ & $1.226(2)$ & $\mathrm{C} 8-\mathrm{H} 8 \mathrm{C}$ & 0.9800 \\
\hline $\mathrm{O} 4-\mathrm{C} 10$ & $1.195(2)$ & $\mathrm{C} 9-\mathrm{C} 10$ & $1.550(3)$ \\
\hline $\mathrm{O} 5-\mathrm{C} 15$ & $1.430(2)$ & $\mathrm{C} 9-\mathrm{C} 12$ & $1.544(2)$ \\
\hline $\mathrm{O} 5-\mathrm{C} 18$ & $1.388(3)$ & $\mathrm{C} 10-\mathrm{C} 11$ & $1.523(3)$ \\
\hline $\mathrm{N} 1-\mathrm{C} 9$ & $1.513(2)$ & $\mathrm{C} 11-\mathrm{C} 13$ & $1.532(3)$ \\
\hline $\mathrm{N} 2-\mathrm{H} 2$ & 0.8800 & $\mathrm{C} 11-\mathrm{C} 17$ & $1.529(3)$ \\
\hline $\mathrm{N} 2-\mathrm{C} 11$ & $1.461(2)$ & $\mathrm{C} 13-\mathrm{H} 13 \mathrm{~A}$ & 0.9900 \\
\hline $\mathrm{N} 2-\mathrm{C} 12$ & $1.325(2)$ & C13-H13B & 0.9900 \\
\hline $\mathrm{C} 1-\mathrm{C} 2$ & $1.407(3)$ & $\mathrm{C} 13-\mathrm{C} 14$ & $1.523(3)$ \\
\hline $\mathrm{C} 1-\mathrm{C} 6$ & $1.390(3)$ & $\mathrm{C} 14-\mathrm{H} 14 \mathrm{~A}$ & 0.9900 \\
\hline $\mathrm{C} 1-\mathrm{C} 9$ & $1.520(3)$ & C14-H14B & 0.9900 \\
\hline $\mathrm{C} 2-\mathrm{C} 3$ & $1.392(3)$ & $\mathrm{C} 14-\mathrm{C} 15$ & $1.500(3)$ \\
\hline $\mathrm{C} 2-\mathrm{C} 7$ & $1.506(3)$ & $\mathrm{C} 15-\mathrm{H} 15$ & 1.0000 \\
\hline $\mathrm{C} 3-\mathrm{H} 3$ & 0.9500 & $\mathrm{C} 15-\mathrm{C} 16$ & $1.518(3)$ \\
\hline $\mathrm{C} 3-\mathrm{C} 4$ & $1.366(4)$ & C16-H16A & 0.9900 \\
\hline $\mathrm{C} 4-\mathrm{H} 4$ & 0.9500 & C16-H16B & 0.9900 \\
\hline $\mathrm{C} 4-\mathrm{C} 5$ & $1.384(4)$ & $\mathrm{C} 16-\mathrm{C} 17$ & $1.525(3)$ \\
\hline $\mathrm{C} 5-\mathrm{C} 6$ & $1.391(3)$ & $\mathrm{C} 17-\mathrm{H} 17 \mathrm{~A}$ & 0.9900 \\
\hline $\mathrm{C} 5-\mathrm{C} 8$ & $1.511(4)$ & C17-H17B & 0.9900 \\
\hline C6- 6 6 & 0.9500 & $\mathrm{C} 18-\mathrm{H} 18 \mathrm{~A}$ & 0.9800 \\
\hline C7-H7A & 0.9800 & C18-H18B & 0.9800 \\
\hline C7-H7B & 0.9800 & $\mathrm{C} 18-\mathrm{H} 18 \mathrm{C}$ & 0.9800 \\
\hline $\mathrm{C} 7-\mathrm{H} 7 \mathrm{C}$ & 0.9800 & & \\
\hline $\mathrm{C} 18-\mathrm{O} 5-\mathrm{C} 15$ & $115.49(19)$ & $\mathrm{N} 2-\mathrm{C} 11-\mathrm{C} 10$ & $101.63(15)$ \\
\hline $\mathrm{O} 1-\mathrm{N} 1-\mathrm{O} 2$ & $124.68(18)$ & $\mathrm{N} 2-\mathrm{C} 11-\mathrm{C} 13$ & $110.96(15)$ \\
\hline $\mathrm{O} 1-\mathrm{N} 1-\mathrm{C} 9$ & $119.64(15)$ & $\mathrm{N} 2-\mathrm{C} 11-\mathrm{C} 17$ & $111.93(15)$ \\
\hline $\mathrm{O} 2-\mathrm{N} 1-\mathrm{C} 9$ & $115.55(16)$ & $\mathrm{C} 10-\mathrm{C} 11-\mathrm{C} 13$ & $110.73(16)$ \\
\hline $\mathrm{C} 11-\mathrm{N} 2-\mathrm{H} 2$ & 121.5 & $\mathrm{C} 10-\mathrm{C} 11-\mathrm{C} 17$ & $111.17(15)$ \\
\hline $\mathrm{C} 12-\mathrm{N} 2-\mathrm{H} 2$ & 121.5 & $\mathrm{C} 17-\mathrm{C} 11-\mathrm{C} 13$ & $110.18(16)$ \\
\hline $\mathrm{C} 12-\mathrm{N} 2-\mathrm{C} 11$ & $117.08(15)$ & $\mathrm{O} 3-\mathrm{C} 12-\mathrm{N} 2$ & $127.30(17)$ \\
\hline $\mathrm{C} 2-\mathrm{C} 1-\mathrm{C} 9$ & $121.12(18)$ & $\mathrm{O} 3-\mathrm{C} 12-\mathrm{C} 9$ & $124.13(16)$ \\
\hline $\mathrm{C} 6-\mathrm{C} 1-\mathrm{C} 2$ & $120.31(18)$ & $\mathrm{N} 2-\mathrm{C} 12-\mathrm{C} 9$ & $108.57(15)$ \\
\hline
\end{tabular}




\begin{tabular}{|c|c|c|c|}
\hline $\mathrm{C} 6-\mathrm{C} 1-\mathrm{C} 9$ & $118.57(17)$ & $\mathrm{C} 11-\mathrm{C} 13-\mathrm{H} 13 \mathrm{~A}$ & 109.3 \\
\hline $\mathrm{C} 1-\mathrm{C} 2-\mathrm{C} 7$ & $125.23(19)$ & $\mathrm{C} 11-\mathrm{C} 13-\mathrm{H} 13 \mathrm{~B}$ & 109.3 \\
\hline $\mathrm{C} 3-\mathrm{C} 2-\mathrm{C} 1$ & $116.0(2)$ & $\mathrm{H} 13 \mathrm{~A}-\mathrm{C} 13-\mathrm{H} 13 \mathrm{~B}$ & 108.0 \\
\hline $\mathrm{C} 3-\mathrm{C} 2-\mathrm{C} 7$ & $118.7(2)$ & $\mathrm{C} 14-\mathrm{C} 13-\mathrm{C} 11$ & $111.56(16)$ \\
\hline $\mathrm{C} 2-\mathrm{C} 3-\mathrm{H} 3$ & 118.4 & $\mathrm{C} 14-\mathrm{C} 13-\mathrm{H} 13 \mathrm{~A}$ & 109.3 \\
\hline $\mathrm{C} 4-\mathrm{C} 3-\mathrm{C} 2$ & $123.2(2)$ & $\mathrm{C} 14-\mathrm{C} 13-\mathrm{H} 13 \mathrm{~B}$ & 109.3 \\
\hline $\mathrm{C} 4-\mathrm{C} 3-\mathrm{H} 3$ & 118.4 & $\mathrm{C} 13-\mathrm{C} 14-\mathrm{H} 14 \mathrm{~A}$ & 109.4 \\
\hline $\mathrm{C} 3-\mathrm{C} 4-\mathrm{H} 4$ & 119.4 & $\mathrm{C} 13-\mathrm{C} 14-\mathrm{H} 14 \mathrm{~B}$ & 109.4 \\
\hline $\mathrm{C} 3-\mathrm{C} 4-\mathrm{C} 5$ & $121.2(2)$ & $\mathrm{H} 14 \mathrm{~A}-\mathrm{C} 14-\mathrm{H} 14 \mathrm{~B}$ & 108.0 \\
\hline $\mathrm{C} 5-\mathrm{C} 4-\mathrm{H} 4$ & 119.4 & $\mathrm{C} 15-\mathrm{C} 14-\mathrm{C} 13$ & $111.35(17)$ \\
\hline $\mathrm{C} 4-\mathrm{C} 5-\mathrm{C} 6$ & $116.8(2)$ & $\mathrm{C} 15-\mathrm{C} 14-\mathrm{H} 14 \mathrm{~A}$ & 109.4 \\
\hline $\mathrm{C} 4-\mathrm{C} 5-\mathrm{C} 8$ & $122.3(2)$ & $\mathrm{C} 15-\mathrm{C} 14-\mathrm{H} 14 \mathrm{~B}$ & 109.4 \\
\hline $\mathrm{C} 6-\mathrm{C} 5-\mathrm{C} 8$ & $121.0(2)$ & $\mathrm{O} 5-\mathrm{C} 15-\mathrm{C} 14$ & $106.08(17)$ \\
\hline $\mathrm{C} 1-\mathrm{C} 6-\mathrm{C} 5$ & $122.4(2)$ & $\mathrm{O} 5-\mathrm{C} 15-\mathrm{H} 15$ & 109.3 \\
\hline $\mathrm{C} 1-\mathrm{C} 6-\mathrm{H} 6$ & 118.8 & $\mathrm{O} 5-\mathrm{C} 15-\mathrm{C} 16$ & $111.55(18)$ \\
\hline $\mathrm{C} 5-\mathrm{C} 6-\mathrm{H} 6$ & 118.8 & $\mathrm{C} 14-\mathrm{C} 15-\mathrm{H} 15$ & 109.3 \\
\hline $\mathrm{C} 2-\mathrm{C} 7-\mathrm{H} 7 \mathrm{~A}$ & 109.5 & $\mathrm{C} 14-\mathrm{C} 15-\mathrm{C} 16$ & $111.32(17)$ \\
\hline $\mathrm{C} 2-\mathrm{C} 7-\mathrm{H} 7 \mathrm{~B}$ & 109.5 & $\mathrm{C} 16-\mathrm{C} 15-\mathrm{H} 15$ & 109.3 \\
\hline $\mathrm{C} 2-\mathrm{C} 7-\mathrm{H} 7 \mathrm{C}$ & 109.5 & $\mathrm{C} 15-\mathrm{C} 16-\mathrm{H} 16 \mathrm{~A}$ & 109.4 \\
\hline $\mathrm{H} 7 \mathrm{~A}-\mathrm{C} 7-\mathrm{H} 7 \mathrm{~B}$ & 109.5 & $\mathrm{C} 15-\mathrm{C} 16-\mathrm{H} 16 \mathrm{~B}$ & 109.4 \\
\hline $\mathrm{H} 7 \mathrm{~A}-\mathrm{C} 7-\mathrm{H} 7 \mathrm{C}$ & 109.5 & $\mathrm{C} 15-\mathrm{C} 16-\mathrm{C} 17$ & $111.29(18)$ \\
\hline $\mathrm{H} 7 \mathrm{~B}-\mathrm{C} 7-\mathrm{H} 7 \mathrm{C}$ & 109.5 & $\mathrm{H} 16 \mathrm{~A}-\mathrm{C} 16-\mathrm{H} 16 \mathrm{~B}$ & 108.0 \\
\hline $\mathrm{C} 5-\mathrm{C} 8-\mathrm{H} 8 \mathrm{~A}$ & 109.5 & $\mathrm{C} 17-\mathrm{C} 16-\mathrm{H} 16 \mathrm{~A}$ & 109.4 \\
\hline $\mathrm{C} 5-\mathrm{C} 8-\mathrm{H} 8 \mathrm{~B}$ & 109.5 & $\mathrm{C} 17-\mathrm{C} 16-\mathrm{H} 16 \mathrm{~B}$ & 109.4 \\
\hline $\mathrm{C} 5-\mathrm{C} 8-\mathrm{H} 8 \mathrm{C}$ & 109.5 & $\mathrm{C} 11-\mathrm{C} 17-\mathrm{H} 17 \mathrm{~A}$ & 109.5 \\
\hline $\mathrm{H} 8 \mathrm{~A}-\mathrm{C} 8-\mathrm{H} 8 \mathrm{~B}$ & 109.5 & $\mathrm{C} 11-\mathrm{C} 17-\mathrm{H} 17 \mathrm{~B}$ & 109.5 \\
\hline $\mathrm{H} 8 \mathrm{~A}-\mathrm{C} 8-\mathrm{H} 8 \mathrm{C}$ & 109.5 & $\mathrm{C} 16-\mathrm{C} 17-\mathrm{C} 11$ & $110.53(16)$ \\
\hline $\mathrm{H} 8 \mathrm{~B}-\mathrm{C} 8-\mathrm{H} 8 \mathrm{C}$ & 109.5 & $\mathrm{C} 16-\mathrm{C} 17-\mathrm{H} 17 \mathrm{~A}$ & 109.5 \\
\hline $\mathrm{N} 1-\mathrm{C} 9-\mathrm{C} 1$ & $112.92(15)$ & $\mathrm{C} 16-\mathrm{C} 17-\mathrm{H} 17 \mathrm{~B}$ & 109.5 \\
\hline $\mathrm{N} 1-\mathrm{C} 9-\mathrm{C} 10$ & $109.82(15)$ & $\mathrm{H} 17 \mathrm{~A}-\mathrm{C} 17-\mathrm{H} 17 \mathrm{~B}$ & 108.1 \\
\hline $\mathrm{N} 1-\mathrm{C} 9-\mathrm{C} 12$ & $104.28(14)$ & $\mathrm{O} 5-\mathrm{C} 18-\mathrm{H} 18 \mathrm{~A}$ & 109.5 \\
\hline $\mathrm{C} 1-\mathrm{C} 9-\mathrm{C} 10$ & $114.16(16)$ & $\mathrm{O} 5-\mathrm{C} 18-\mathrm{H} 18 \mathrm{~B}$ & 109.5 \\
\hline $\mathrm{C} 1-\mathrm{C} 9-\mathrm{C} 12$ & $113.32(15)$ & $\mathrm{O} 5-\mathrm{C} 18-\mathrm{H} 18 \mathrm{C}$ & 109.5 \\
\hline $\mathrm{C} 12-\mathrm{C} 9-\mathrm{C} 10$ & $101.33(14)$ & $\mathrm{H} 18 \mathrm{~A}-\mathrm{C} 18-\mathrm{H} 18 \mathrm{~B}$ & 109.5 \\
\hline $\mathrm{O} 4-\mathrm{C} 10-\mathrm{C} 9$ & $127.03(18)$ & $\mathrm{H} 18 \mathrm{~A}-\mathrm{C} 18-\mathrm{H} 18 \mathrm{C}$ & 109.5 \\
\hline $\mathrm{O} 4-\mathrm{C} 10-\mathrm{C} 11$ & $124.44(18)$ & $\mathrm{H} 18 \mathrm{~B}-\mathrm{C} 18-\mathrm{H} 18 \mathrm{C}$ & 109.5 \\
\hline $\mathrm{C} 11-\mathrm{C} 10-\mathrm{C} 9$ & $108.53(15)$ & & \\
\hline $\mathrm{O} 1-\mathrm{N} 1-\mathrm{C} 9-\mathrm{C} 1$ & $-18.9(2)$ & $\mathrm{C} 6-\mathrm{C} 1-\mathrm{C} 9-\mathrm{N} 1$ & $120.84(19)$ \\
\hline $\mathrm{O} 1-\mathrm{N} 1-\mathrm{C} 9-\mathrm{C} 10$ & $-147.60(16)$ & $\mathrm{C} 6-\mathrm{C} 1-\mathrm{C} 9-\mathrm{C} 10$ & $-112.8(2)$ \\
\hline $\mathrm{O} 1-\mathrm{N} 1-\mathrm{C} 9-\mathrm{C} 12$ & $104.50(18)$ & $\mathrm{C} 6-\mathrm{C} 1-\mathrm{C} 9-\mathrm{C} 12$ & $2.6(2)$ \\
\hline $\mathrm{O} 2-\mathrm{N} 1-\mathrm{C} 9-\mathrm{C} 1$ & $165.05(16)$ & $\mathrm{C} 7-\mathrm{C} 2-\mathrm{C} 3-\mathrm{C} 4$ & $176.7(3)$ \\
\hline $\mathrm{O} 2-\mathrm{N} 1-\mathrm{C} 9-\mathrm{C} 10$ & $36.4(2)$ & $\mathrm{C} 8-\mathrm{C} 5-\mathrm{C} 6-\mathrm{C} 1$ & $177.3(2)$ \\
\hline $\mathrm{O} 2-\mathrm{N} 1-\mathrm{C} 9-\mathrm{C} 12$ & $-71.51(19)$ & $\mathrm{C} 9-\mathrm{C} 1-\mathrm{C} 2-\mathrm{C} 3$ & $-178.3(2)$ \\
\hline $\mathrm{O} 4-\mathrm{C} 10-\mathrm{C} 11-\mathrm{N} 2$ & $-167.1(2)$ & $\mathrm{C} 9-\mathrm{C} 1-\mathrm{C} 2-\mathrm{C} 7$ & $4.9(3)$ \\
\hline $\mathrm{O} 4-\mathrm{C} 10-\mathrm{C} 11-\mathrm{C} 13$ & $-49.2(3)$ & $\mathrm{C} 9-\mathrm{C} 1-\mathrm{C} 6-\mathrm{C} 5$ & $179.1(2)$ \\
\hline $\mathrm{O} 4-\mathrm{C} 10-\mathrm{C} 11-\mathrm{C} 17$ & $73.6(3)$ & $\mathrm{C} 9-\mathrm{C} 10-\mathrm{C} 11-\mathrm{N} 2$ & $12.77(19)$ \\
\hline $\mathrm{O} 5-\mathrm{C} 15-\mathrm{C} 16-\mathrm{C} 17$ & $-174.73(17)$ & $\mathrm{C} 9-\mathrm{C} 10-\mathrm{C} 11-\mathrm{C} 13$ & $130.69(17)$ \\
\hline
\end{tabular}




$\begin{array}{llll}\mathrm{N} 1-\mathrm{C} 9-\mathrm{C} 10-\mathrm{O} 4 & 53.2(3) & \mathrm{C} 9-\mathrm{C} 10-\mathrm{C} 11-\mathrm{C} 17 & -106.48(18) \\ \mathrm{N} 1-\mathrm{C} 9-\mathrm{C} 10-\mathrm{C} 11 & -126.63(16) & \mathrm{C} 10-\mathrm{C} 9-\mathrm{C} 12-\mathrm{O} 3 & -164.71(18) \\ \mathrm{N} 1-\mathrm{C} 9-\mathrm{C} 12-\mathrm{O} 3 & -50.6(2) & \mathrm{C} 10-\mathrm{C} 9-\mathrm{C} 12-\mathrm{N} 2 & 14.88(19) \\ \mathrm{N} 1-\mathrm{C} 9-\mathrm{C} 12-\mathrm{N} 2 & 128.95(15) & \mathrm{C} 10-\mathrm{C} 11-\mathrm{C} 13-\mathrm{C} 14 & 178.93(18) \\ \mathrm{N} 2-\mathrm{C} 11-\mathrm{C} 13-\mathrm{C} 14 & -69.0(2) & \mathrm{C} 10-\mathrm{C} 11-\mathrm{C} 17-\mathrm{C} 16 & -179.12(16) \\ \mathrm{N} 2-\mathrm{C} 11-\mathrm{C} 17-\mathrm{C} 16 & 68.0(2) & \mathrm{C} 11-\mathrm{N} 2-\mathrm{C} 12-\mathrm{O} 3 & 171.67(18) \\ \mathrm{C} 1-\mathrm{C} 2-\mathrm{C} 3-\mathrm{C} 4 & -0.4(4) & \mathrm{C} 11-\mathrm{N} 2-\mathrm{C} 12-\mathrm{C} 9 & -7.9(2) \\ \mathrm{C} 1-\mathrm{C} 9-\mathrm{C} 10-\mathrm{O} 4 & -74.7(3) & \mathrm{C} 11-\mathrm{C} 13-\mathrm{C} 14-\mathrm{C} 15 & -55.5(2) \\ \mathrm{C} 1-\mathrm{C} 9-\mathrm{C} 10-\mathrm{C} 11 & 105.40(18) & \mathrm{C} 12-\mathrm{N} 2-\mathrm{C} 11-\mathrm{C} 10 & -3.1(2) \\ \mathrm{C} 1-\mathrm{C} 9-\mathrm{C} 12-\mathrm{O} 3 & 72.5(2) & \mathrm{C} 12-\mathrm{N} 2-\mathrm{C} 11-\mathrm{C} 13 & -120.87(18) \\ \mathrm{C} 1-\mathrm{C} 9-\mathrm{C} 12-\mathrm{N} 2 & -107.87(17) & \mathrm{C} 12-\mathrm{N} 2-\mathrm{C} 11-\mathrm{C} 17 & 115.59(18) \\ \mathrm{C} 2-\mathrm{C} 1-\mathrm{C} 6-\mathrm{C} 5 & -0.3(3) & \mathrm{C} 12-\mathrm{C} 9-\mathrm{C} 10-\mathrm{O} 4 & 163.1(2) \\ \mathrm{C} 2-\mathrm{C} 1-\mathrm{C} 9-\mathrm{N} 1 & -59.8(2) & \mathrm{C} 12-\mathrm{C} 9-\mathrm{C} 10-\mathrm{C} 11 & -16.77(19) \\ \mathrm{C} 2-\mathrm{C} 1-\mathrm{C} 9-\mathrm{C} 10 & 66.6(2) & \mathrm{C} 13-\mathrm{C} 11-\mathrm{C} 17-\mathrm{C} 16 & -56.0(2) \\ \mathrm{C} 2-\mathrm{C} 1-\mathrm{C} 9-\mathrm{C} 12 & -178.06(18) & \mathrm{C} 13-\mathrm{C} 14-\mathrm{C} 15-\mathrm{O} 5 & 176.97(17) \\ \mathrm{C} 2-\mathrm{C} 3-\mathrm{C} 4-\mathrm{C} 5 & -1.3(4) & \mathrm{C} 13-\mathrm{C} 14-\mathrm{C} 15-\mathrm{C} 16 & 55.5(2) \\ \mathrm{C} 3-\mathrm{C} 4-\mathrm{C} 5-\mathrm{C} 6 & 2.1(4) & \mathrm{C} 14-\mathrm{C} 15-\mathrm{C} 16-\mathrm{C} 17 & -56.5(2) \\ \mathrm{C} 3-\mathrm{C} 4-\mathrm{C} 5-\mathrm{C} 8 & -176.6(3) & \mathrm{C} 15-\mathrm{C} 16-\mathrm{C} 17-\mathrm{C} 11 & 56.7(2) \\ \mathrm{C} 4-\mathrm{C} 5-\mathrm{C} 6-\mathrm{C} 1 & -1.3(3) & \mathrm{C} 17-\mathrm{C} 11-\mathrm{C} 13-\mathrm{C} 14 & 55.5(2) \\ \mathrm{C} 6-\mathrm{C} 1-\mathrm{C} 2-\mathrm{C} 3 & 1.1(3) & \mathrm{C} 18-\mathrm{O} 5-\mathrm{C} 15-\mathrm{C} 14 & 150.9(2) \\ \mathrm{C} 6-\mathrm{C} 1-\mathrm{C} 2-\mathrm{C} 7 & -175.7(2) & \mathrm{C} 18-\mathrm{O} 5-\mathrm{C} 15-\mathrm{C} 16 & -87.7(2) \\ & & & \end{array}$

Hydrogen-bond geometry $\left(\AA,{ }^{\circ}\right)$

\begin{tabular}{lllll}
\hline$D-\mathrm{H} \cdots A$ & $D-\mathrm{H}$ & $\mathrm{H} \cdots A$ & $D \cdots A$ & $D-\mathrm{H}^{\cdots} A$ \\
\hline $\mathrm{N} 2-\mathrm{H} 2 \cdots \mathrm{O} 3^{\mathrm{i}}$ & 0.88 & 2.02 & $2.8853(19)$ & 167 \\
$\mathrm{C} 4-\mathrm{H} 4 \cdots 5^{\mathrm{ii}}$ & 0.95 & 2.57 & $3.287(3)$ & 132 \\
$\mathrm{C} 7-\mathrm{H} 7 B \cdots \mathrm{O} 1^{\mathrm{iii}}$ & 0.98 & 2.49 & $3.454(3)$ & 168 \\
$\mathrm{C} 14-\mathrm{H} 14 B \cdots \mathrm{O} 2^{\mathrm{iv}}$ & 0.99 & 2.54 & $3.265(3)$ & 130
\end{tabular}

Symmetry codes: (i) $-x+1,-y+2,-z+1$; (ii) $x,-y+3 / 2, z-1 / 2$; (iii) $-x, y-1 / 2,-z+1 / 2$; (iv) $-x,-y+2,-z+1$. 